\title{
Cardiac arrest in pregnancy and perimortem cesarean delivery: case report and discussion
}

\author{
Paul T. Engels, MD* ; Sheila C. Caddy, MD; Gulnaz Jiwa, MD; J. Douglas Matheson, MD ${ }^{\dagger \ddagger}$
}

\section{ABSTRACT}

Cardiac arrest in pregnancy is a rare occurrence, particularly in the emergency department setting. The resuscitation of a pregnant patient in cardiac arrest is unique in a number of ways. Early identification and treatment of possible etiologies, appropriate response to the physiologic changes present in pregnancy, relief of potential vena cava obstruction by the gravid uterus, and expeditious preparation for possible cesarean delivery are important considerations for a successful resuscitation. We report and discuss the case of a pregnant patient with pulmonary edema and cardiac dysfunction who presented with severe hypoxemia and subsequent cardiac arrest and underwent a perimortem cesarean delivery and simultaneous fetal and maternal resuscitation in the emergency department.

\section{RÉSUMÉ}

L'arrêt cardiaque chez les femmes enceintes est rare, en particulier dans le cadre des urgences. La réanimation des patientes enceintes ayant subi un arrêt cardiaque est unique à plus d'un égard. L'identification et le traitement rapides des étiologies possibles, la réponse convenable aux changements physiologiques que présentent les patientes enceintes, le soulagement d'une obstruction possible de la veine profonde par l'utérus gravide et la préparation rapide d'un accouchement possible par césarienne sont des facteurs importants qui contribuent au succès des efforts de réanimation. Nous rapportons le cas d'une patiente enceinte atteinte d'un œdème pulmonaire et d'une dysfonction cardiaque qui présentait une hypoxémie aiguë, suivie d'un arrêt cardiaque, et qui a subi un accouchement par césarienne peri-mortem ainsi qu'une réanimation simultanée du fœtus et de la mère aux urgences, et en discutons.
Keywords: cardiac arrest, cardiopulmonary resuscitation, cesarean section, critical care, emergency department, emergency medicine, pregnancy

Cardiac arrest in a pregnant female is a rare but terrifying situation, with an incidence estimated to be 1 in 30,000 pregnancies. ${ }^{1}$ To place this in perspective, there were approximately 377,000 births in 2008-2009 in Canada, including 51,000 in Alberta. ${ }^{2}$ Thus, a province such as Alberta may have one or two such events per year. Maternal collapse, although less well defined and documented, is likely far more common and generates similar challenges in its management. ${ }^{3}$ Important differences exist specific to resuscitating a pregnant patient, including altered respiratory mechanics and decreased reserve, an enlarged uterus that can cause aortocaval compression, and swollen tissues with the potential for challenges in airway management. Management of this difficult scenario requires expeditious and decisive treatment targeted at maternal resuscitation, the identification of any readily correctable etiologies, and preparation for perimortem cesarean delivery.

\section{CASE REPORT}

A previously healthy 23-year-old pregnant female, gravida 1, para 0 at $37+$ weeks gestational age, was sent to the emergency department (ED) of a community hospital in Alberta after her primary care physician found her to have a blood pressure of 150/105 $\mathrm{mm} \mathrm{Hg}$. Three weeks previously, she had been started on oral labetolol after hypertension had been identified. In the

Division of Critical Care Medicine, University of Alberta Hospital; †Intensive Care Unit, Royal Alexandra Hospital; ¥Department of Surgery, Royal Alexandra Hospital; §Department of Obstetrics and Gynecology, Royal Alexandra Hospital, Edmonton, AB.

Correspondence to: Dr. Paul Engels, Division of Critical Care Medicine, Room 3C1.12, Walter C. Mackenzie Centre, University of Alberta Hospital, 8440-112 Street, Edmonton, AB T6G 2B7; pengels@ualberta.ca.

Submitted May 2, 2010; Revised August 13 and 25, 2010; Accepted September 2, 2010.

This article has been peer reviewed. 
ED, her initial vital signs were heart rate 130 beats/ min, blood pressure $148 / 88 \mathrm{~mm} \mathrm{Hg}$, respiratory rate 22 breaths/min, temperature $36.0^{\circ} \mathrm{C}\left(96.8^{\circ} \mathrm{F}\right)$, and oxygen saturation $88 \%$ on room air. Oxygen was applied by nasal cannula, which improved the patient's oxygen saturation to $95 \%$. Chest radiography was performed and interpreted by her primary care physician as showing pneumonia, although the subsequent radiologist's interpretation reported "bilateral extensive airspace opacification highly suspicious for pulmonary edema." Blood investigations revealed hemoglobin $117 \mathrm{~g} / \mathrm{L}$, white blood count $18.2 \times 10^{9} / \mathrm{L}$, platelets $435 \times 10^{9} / \mathrm{L}$, and alanine transaminase $7 \mathrm{IU} / \mathrm{L}$, and urinalysis showed $3+$ proteinuria.

She was admitted to the hospital under the care of her primary care physician and was administered antibiotics for the presumptive diagnosis of pneumonia and analgesics. Through the night, she had significant orthopnea and a respiratory rate of 40 to 50 breaths/ min. The next morning, her oxygen saturation was $93 \%$ on $15 \mathrm{~L} / \mathrm{min}_{2}$ by a nonrebreather mask. Her physician made arrangements with an obstetrician at an obstetrical referral hospital in Edmonton, $40 \mathrm{~km}$ away, for patient transfer via Advanced Life Support Ambulance.

During transport, the patient's oxygen saturation deteriorated to less than $80 \%$, and the ambulance was redirected to the nearest hospital, which happened to be the regional tertiary care obstetric hospital and a level II trauma centre. On arrival in the ED, the patient was in extremis, with a heart rate over 120 beats $/ \mathrm{min}$, blood pressure $180 / 90 \mathrm{~mm} \mathrm{Hg}$, and oxygen saturation of $45 \%$. She was placed in the supine position and orotracheally intubated in rapid sequence fashion with propofol and succinylcholine. Immediately following intubation, the patient became bradycardic, with no palpable pulses. Cardiopulmonary resuscitation (CPR) was commenced following an Advanced Cardiac Life Support (ACLS) algorithm for pulseless electrical activity. Over a 5 -minute period of supine CPR, the patient remained in bradycardic pulseless electrical activity and had received a total of $2 \mathrm{mg}$ of epinephrine, $2 \mathrm{mg}$ of atropine, and 1 ampule of sodium bicarbonate; however, return of spontaneous circulation was not achieved.

At this point, the decision was made to perform a perimortem cesarean delivery. After a slight delay to obtain an appropriate scalpel blade and handle and Kelly clamps (the only instruments used), a cesarean delivery was performed using a vertical midline incision and vertical hysterotomy by the intensive care unit (ICU) team consisting of two general surgeons and an obstetrics resident. The uterus was packed with sponges following fetal and placental delivery. CPR was continued throughout this procedure, and the fetus was delivered approximately 8 minutes after the maternal cardiac arrest. Resuscitation of the mother continued, and after 30 minutes, return of spontaneous circulation was achieved. A chest radiograph showed diffuse bilateral air-space opacification. The infant was resuscitated in the emergency ED by the neonatal intensive care unit (NICU) team and required 10 minutes of $\mathrm{CPR}$ before spontaneous circulation was achieved and transfer to the on-site level III nursery could proceed.

The mother was taken emergently to the operating room for closure of the hysterotomy and laparotomy. An intraoperative echocardiogram revealed severe biventricular dysfunction, and the patient was transferred to the cardiac angiography laboratory for placement of an intra-aortic balloon pump before admission to the ICU. In the ICU, the patient required a fraction of inspired oxygen $\left(\mathrm{F}_{\mathrm{i}} \mathrm{O}_{2}\right)$ of $100 \%$, positive end-expiratory pressure of $20 \mathrm{~cm} \mathrm{H}_{2} \mathrm{O}$, and high-dose infusions of vasopressin, norepinephrine, epinephrine, dobutamine, and milrinone. She was anuric, and continuous renal replacement therapy was initiated. Her clinical picture was consistent with severe preeclampsia resulting in pulmonary edema and cardiac dysfunction. Over the next 7 days, the patient's oxygen requirements decreased; she was weaned off inotropes and vasopressors, and her renal function recovered. Unfortunately, it was evident that she had suffered a severe hypoxic-ischemic neurologic injury that would likely result in a persistent vegetative state. After discussions with her family, the patient's medical support was limited, and she subsequently died 11 days after her cardiac arrest.

The infant was severely acidotic and hypoxemic on delivery, with a first recorded $\mathrm{pH}$ of 6.8 , and required major respiratory and hemodynamic support in the NICU. She developed intractable seizures secondary to severe anoxic brain injury and died 18 hours after delivery after support was withdrawn.

\section{DISCUSSION}

The etiology of cardiac arrest in a pregnant female includes both general causes and, most importantly, those specific to, or with an elevated likelihood from, the gravid state (Table 1). The clinical suspicion for 
Table 1. Causes of cardiac arrest during pregnancy

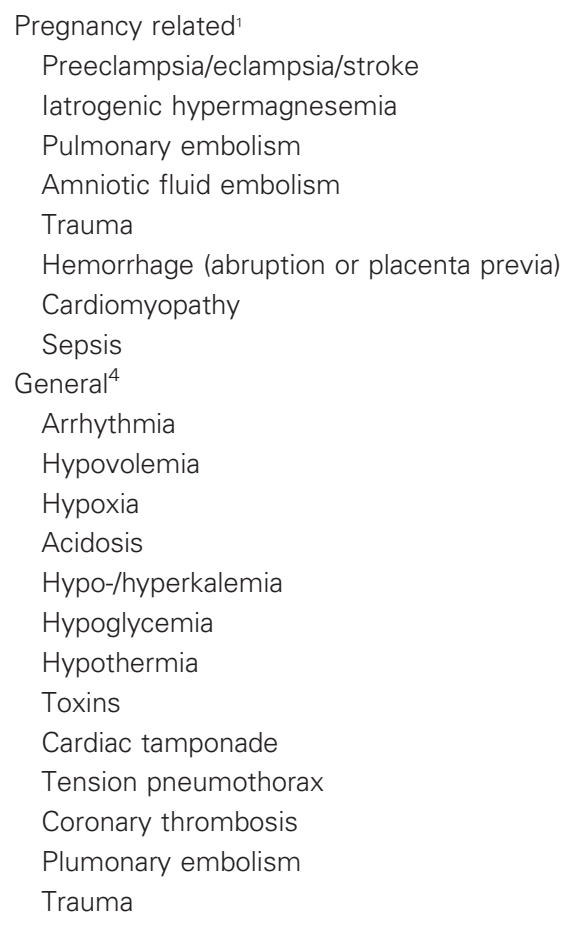

each of these etiologies will vary with the antecedent history and context of care. For example, in a pregnant patient undergoing elective cesarean section who has a cardiac arrest as the obstetrician is manipulating the uterus, one should have a high suspicion for an amniotic fluid embolism; alternatively, in a patient who has a cardiac arrest while on an infusion of $\mathrm{MgSO}_{4}$ for preeclampsia, one should have a high suspicion for either eclampsia or magnesium toxicity as the cause.

The key reason for identifying a likely etiology for the cardiac arrest is to determine if any specific therapeutic options can be instituted. In a pregnant patient with cardiac arrest, both standard resuscitation and consideration and preparation for possible perimortem cesarean section should begin immediately.

Resuscitation of the pregnant patient who has a cardiac arrest should follow the American Heart Association (AHA) guidelines for adults, ${ }^{4}$ with a few alterations specific for the gravid state. Figure 1 provides a flow diagram of the general management principles, and the following should be considered:

- In a gestational age $>20$ weeks (ie, uterine fundus at the umbilicus or higher), shift the uterus away from the inferior vena cava (IVC) by placing the patient 15 to $30^{\circ}$ in the left lateral position or by pulling the uterus to the side. This can be achieved by inserting a wedge or rolled blanket under the patient's right hip.

- Perform chest compressions slightly above the centre of the sternum (higher than normal).

- Secure the airway early (do not wait for the completion of one cycle of chest compressions).

- Do not obtain subdiaphragmatic venous access as the uterus may compress the IVC and prevent drugs

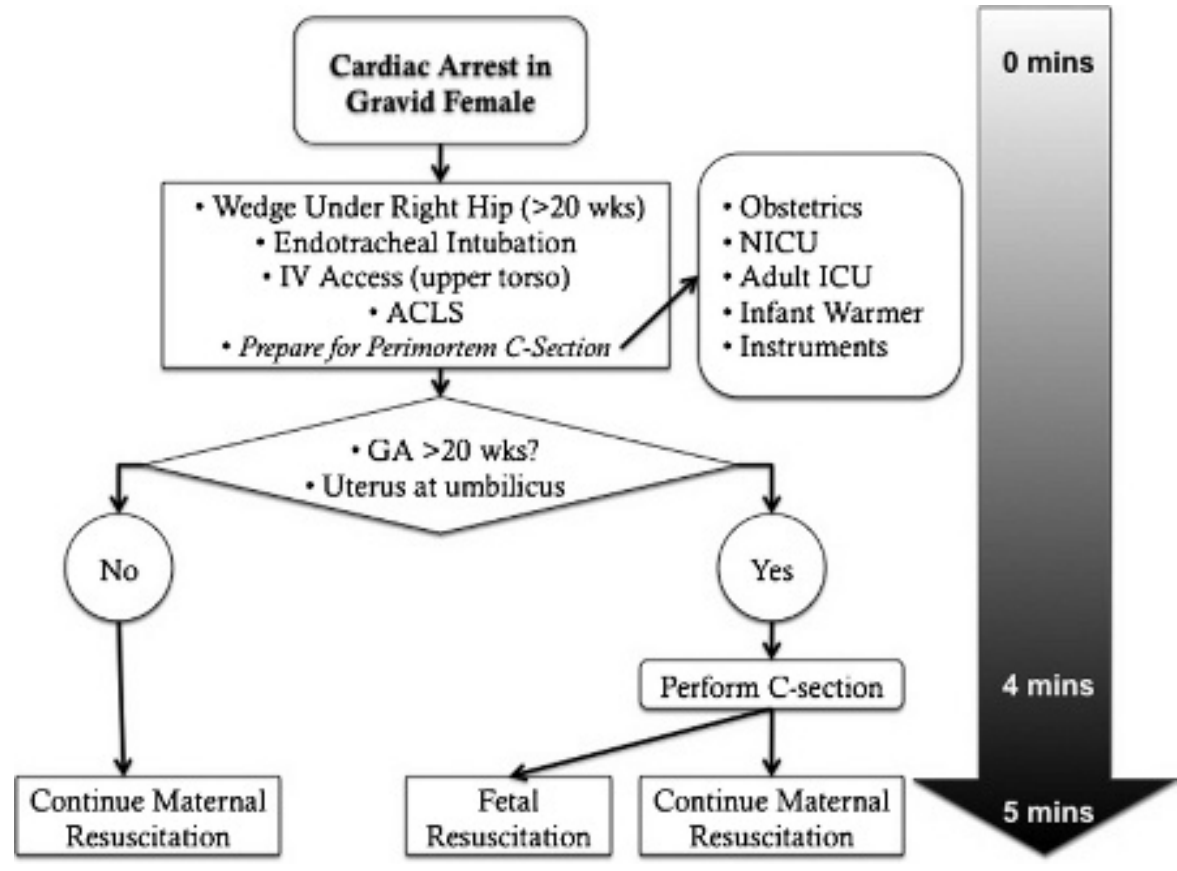

Figure 1. Management of cardiac arrest in a pregnant patient. ACLS = Advanced Cardiac Life Support; Csection $=$ cesarean section; $\mathrm{GA}=$ gestational age; ICU = intensive care unit; IV = intravenous; NICU = neonatal intensive care unit. 
from reaching the heart. Obtain upper limb peripheral intravenous (IV) lines or central venous access via the internal jugular or subclavian veins.

- Defibrillate using standard doses; do not alter the dose or pad placement.

- Use standard ACLS medications and administration guidelines; do not alter the dose or frequency.

- Begin considering emergency hysterotomy/perimortem cesarean delivery early in the resuscitation efforts.

Emergency hysterotomy, or perimortem cesarean delivery, is the expeditious surgical delivery of a fetus while the mother is in a state of cardiac arrest. Historically, this was used as a procedure of last resort to attempt "fetal salvage" after failure of maternal resuscitation. In the 1980 s, reports of maternal recovery after fetal delivery were published. In 1986, the literature on this topic was reviewed and summarized by Katz and colleagues.5 They found 269 cases with 188 infant survivals after reviewing all reported cases since 1879 and, based on an analysis of fetal outcomes stratified by delivery time, proposed a "Four-Minute-Rule" whereby "cesarean delivery should be begun within four minutes and the infant delivered within five minutes after maternal cardiac arrest." ${ }_{5}$ In 2005, almost 20 years after their recommendations were published, Katz and colleagues again reviewed the literature and identified an additional 38 cases with outcomes that supported the Four-MinuteRule. ${ }^{6}$ They also found that there was return of spontaneous circulation or improvement in hemodynamic status in 12 of 20 perimortem cesarean deliveries that reported maternal outcome. Physiologically, evacuation of the gravid uterus has a number of beneficial maternal effects: it (1) alleviates IVC compression and thereby improves venous return; (2) allows for more mechanically effective CPR; and (3) allows redistribution of the uterine blood flow (up to $25 \%$ of cardiac output at term) to the other organs. Based on their reassessment of the literature, Katz and colleagues recommended "for any indication of maternal cardiac arrest, if pulses cannot be obtained, the cesarean delivery should be performed." $"$ The current AHA guidelines advise that for a gestational age of $>$ 20 weeks, a perimortem cesarean section should be performed. ${ }^{4}$ The International Liaison Committee on Resuscitation published its consensus guidelines in Circulation in October 2010 and reaffirmed these recommendations. ${ }^{7}$

Although standard supportive care and resuscitation are the norm, a few etiologies of cardiac arrest in pregnancy exist for which specific treatment can be lifesaving (Table 2). Of note, thrombolysis is no longer contraindicated during pregnancy. A Medline keyword search of "thrombolysis AND pregnancy" restricted to English articles revealed a number of case reports and a recent review of massive pulmonary embolism during pregnancy by te Raa and colleagues. ${ }^{8}$ They identified 13 pregnant patients who received thrombolysis with no maternal deaths, two fetal deaths, and five preterm deliveries and concluded that "current data do not justify withholding pregnant women from thrombolytic therapy in case of life-threatening pulmonary embolism."

In our case, a number of important learning points exist. Understanding the presentation, diagnosis, and acute deterioration potential of patients with preeclampsia is mandatory for primary care, emergency, and obstetrics practitioners, and early consultation with a local referral centre is encouraged. The responsibility for providing safe patient transfer between facilities is shared between the sending and receiving physicians, and any concern about the safety of such a transfer should prompt the involvement of critical care resources. Pregnant patients present many unique challenges in their resuscitation, including hip wedge, appropriate IV access, and a difficult airway, and the need to emergently intubate such patients should trigger the early involvement of airway and resuscitation experts. Creation of a dedicated perimortem cesarean equipment pack, and its ready location within the ED, has been recommended previously ${ }^{9}$ and would have improved the time to delivery in our case.

\begin{tabular}{|l|}
\hline Table 2. Potentially treatable causes of cardiac arrest in pregnancy \\
\hline Etiology
\end{tabular}


A recent report from the United Kingdom recommended improvement in the level of advanced life support skills for pregnant patients by clinical staff. ${ }^{10}$ Courses such as Advanced Life Support in Obstetrics (ALSO), Managing Obstetric Emergencies \& Trauma (MOET), and Advances in Labour and Risk Management (ALARM), as well as hospital-based simulations, have the potential to improve the care delivered in such dire situations. Practitoners in centres that care for pregnant patients may face the need to perform a perimortem cesarean delivery, and a consideration of these learning points has the potential to improve the outcome of such an occurrence.

\section{CONCLUSION}

When confronted with a pregnant patient who suffers a cardiac arrest, it is important to act quickly and decisively while also keeping in mind the unique priorities of this rare situation. The first priorities are ACLS, early securing of the airway, and, in any pregnant woman with a uterus at the umbilicus or higher, placement of a 15 to $30^{\circ}$ wedge under the patient's right hip. Second, immediate consultation with Obstetrics, the adult ICU, and the NICU are essential, as is the preparation for perimortem ceserean delivery. Third, a rapid assessment of the potential etiology of the patient's arrest is important and may provide clues about specific treatment that could be instituted. Finally, if the gestation age is $>20$ weeks and return of maternal spontaneous circulation has not been achieved within 4 minutes, cesarean delivery should be initiated if the centre and practitioners involved have such capabilities. The probability of maternal and fetal survival is optimized by the successful achievement of all of these priorities within this extremely narrow timeframe. To achieve this, the rapid response of a coordinated team that is knowledgeable regarding the priorities of resuscitation as they relate to maternal collapse, readily available supplies, and expertise are essential. Ensuring that these components are available and functioning, through preparation and training, will allow practitioners to properly manage such an extraordinary event and give their patients the best chance for a good outcome.

Competing interests: None declared.

\section{REFERENCES}

1. Atta E, Gardner M. Cardiopulmonary resucitation in pregnancy. Obstet Gynecol Clin N Am 2007;34:585-97.

2. Statistics Canada. Births, estimates, by province and territory. Available at: http://www40.statcan.ca/101/cst01/demo04a-eng. htm (accessed December 14, 2009).

3. McDonnell NJ. Preventing maternal morbidity and mortality: management of the collapsed obstetric patient. In: Australasian Anaesthesia 2009: invited papers and selected continuing education lectures. Available at: http://www.anzca.edu. au/resources/books-and-publications/ANZCA_Australasian_ Anaesthesia_09.pdf (accessed August 4, 2010).

4. 2005 American Heart Association guidelines for cardiopulmonary resuscitation and emergency cardiovascular care. Circulation 2005;112(24 Suppl):IV1-203.

5. Katz V, Dotters DJ, Droegemueller W. Perimortem cesarean delivery. Obstet Gynecol 1986;68:571.

6. Katz V, Balderston K, DeFreest M. Perimortem cesarean delivery: were our assumptions correct? Am 7 Obstet Gynecol 2005;192:1916-21.

7. Vanden Hoek TL, Morrison LJ, Shuster M, et al. Part 12: cardiac arrest in special situations: 2010 American Heart Association guidelines for cardiopulmonary resuscitation and emergency cardiovascular care. Circulation 2010;122:S829861.

8. te Raa GD, Ribbert LSM, Snijder RJ, et al. Treatment options in massive pulmonary embolism during pregnancy: a case-report and review of literature. Thromb Res 2009;124: $1-5$.

9. McDonnell NJ. Cardiopulmonary arrest in pregnancy: two case reports of successful outcomes in association with perimortem caesarean delivery. Br 7 Anaesth 2009;103:406-9.

10. Lewis G, editor. The Confidential Enquiry into Maternal and Child Health (CEMACH). Saving mothers' lives: reviewing maternal deaths to make motherbood safer 2003-2005. The seventh report on Confidential Enquiries into Maternal Deatbs in the United Kingdom. London: CEMACH; 2007. 